\title{
Selective spraying of grapevine's diseases by a modular agricultural robot
}

\author{
R. Oberti, ${ }^{1}$ M. Marchi, ${ }^{1,2}$ P. Tirelli, ${ }^{1,2}$ A. Calcante, ${ }^{1}$ M. Iriti, ${ }^{1}$ M. Hočevar, ${ }^{3}$ J. Baur, ${ }^{4}$ \\ J. Pfaff, ${ }^{4}$ C. Schütz, ${ }^{4}$ H. Ulbrich ${ }^{4}$ \\ ${ }^{1}$ DiSAA, Dipartimento di Scienze Agrarie e Ambientali, Università degli Studi di Milano, Italy, \\ ${ }^{2}$ Applied Intelligent Systems-AIS Lab, Dipartimento di Informatica, Università degli Studi di \\ Milano, Italy; ${ }^{3}$ University of Ljubljana, Faculty of Mechanical Engineering, Ljubljana, Slovenia; \\ ${ }^{4}$ Institute of Applied Mechanics, Technische Universität München, Garching, Germany
}

\begin{abstract}
In current viticulture protection of grapevine is obtained with uniform distribution of fungicides, typically repeated according a regular calendar. This continuous protection approach can easily result in ten to fifteen treatments per season in vineyards of several wine-producing regions. Primary infections exhibit nevertheless discrete foci, with uneven spatial distribution. Hence it can be argued that detection of symptoms at early disease stages and their targeted treatment would reduce the spread of the infection to wider patches in the vineyard, while enabling reduced use of pesticides. Within the UE-project CROPS, a modular and multifunctional agricultural robot system for specialty crops is being developed and one of the tasks that has to accomplished is selective spraying of diseases. The robotic system setup integrates a six degrees of freedom manipulator, an optical sensor system and a precision spraying actuator. After a brief description of the requirements of the system, this contribution gives a detailed description of its components and discusses the results obtained in first experiments. As case study we consider here the automatic detection and selective spraying of grapevine canopy areas exhibiting symptoms of powdery mildew (Erysiphe necator), one of the major diseases for this crop. Based on optical sensing feedback, the precision spraying actuator is positioned by the manipulator to selectively and accurately apply pesticides solely to infected areas.

Disease foci identification and localization is based on on-the-go processing of images sensed by a multispectral camera inspecting the
\end{abstract}

Correspondence: Roberto Oberti, DiSAA, Dipartimento di Scienze Agrarie e Ambientali, Università degli Studi di Milano, via Celoria 2 - Milano, Italy. E-mail: roberto.oberti@unimi.it

Key words: Precision spraying , agricultural robot, crop protection, automation, disease sesning.

Acknowledgements: CROPS (GA-246252) is funded by the European Commission under the 7th Framework Programme within the theme "Automation and robotics for sustainable crop and forestry management"

(C) Copyright R. Oberti et al., 2013

Licensee PAGEPress, Italy

Journal of Agricultural Engineering 2013; XLIV(s2):e29

doi:10.4081/jae.2013.s2.e29

This article is distributed under the terms of the Creative Commons Attribution Noncommercial License (by-nc 3.0) which permits any noncommercial use, distribution, and reproduction in any medium, provided the original author(s) and source are credited. vertical structure of the grapevine canopy. At the end of the manipulator arm is located the precision spraying actuator, constituted by an axial fan with a flow straightener and an axially mounted spraying nozzle. The sprayer can deliver an air-carrier flow with an adjustable velocity, producing a circular spraying pattern of a constant diameter of $0.15 \mathrm{~m}$ over a wide range of spraying distances.

A first experiment was conducted in an experimental greenhouse, where vineyard canopy conditions were recreated by aligning plants of grapevine grown in pots. Within the recreated canopy, diseased plants with different levels of disease symptoms were used as targets of automated selective spraying performed by the agricultural robot. The results of these experiments are discussed in view of a possible intelligent, close precision crop protection framework.

\section{Introduction}

In current farming practice, pesticides are typically applied uniformly to the fields. This, despite several pests and diseases exhibit an uneven spatial distribution, with typical patch structures evolving around discrete foci, especially during early stages of development. Grapevine is not an exception and, in current viticulture practice, fungicides are applied uniformly through the vineyard according a spraying calendar, commonly based on regular and frequent fungicide applications, more rarely triggered by experts decisions or objective data. For powdery mildew (Erysiphe necator) and downy mildew (Plasmopora viticola), two major grapevine fungal diseases, this continuous protection approach can easily result in ten to fifteen treatments per season, often at application rates of $500-1000 \mathrm{dm}^{3} / \mathrm{ha}$ each, for many vineyards in some of the most advanced wine-producing regions worldwide.

Pesticides are recognised to play a major role in environmental pressure and production costs of agricultural activity, as well as in public concerns about healthiness and wholesomeness products. There is then an increasing interest in developing suitable techniques and equipment able to selectively target the application of pesticides where and when needed by the crop, with the aim of preventing or inhibiting the establishment of the infection and its epidemic spread to the whole field.

Growing labor costs and unavailability of skilled personnel during work-peak periods in most European countries, promote the research on advanced automation for selective agricultural cultivation processes. Since growing of crops requires several operations with a huge variety of combinations in parameters, only a highly modular and reconfigurable robotic system suitable for different specialty crops (grapes, sweet-pepper, apples) as well as for multiple tasks (spraying, selective harvesting), can achieve high utilization grades.

The main task of UE project CROPS (www.crops-robots.eu) is to 
develop, optimize and demonstrate a multipurpose, modular and lightweight manipulator able to cope with these specific requirements. The adopted approach it's clearly distinguished from that of other research groups which use non-modular and heavy standard industrial manipulators (see e.g. Baeten et al., 2008; Katupitiya et al., 2008) or which focus on one specific fruit and purpose (see e.g. Guo et al., 2010; Kitamura/Oka, 2005).

One of the challenging applications of the new CROPS manipulator is the selective, intelligent targeting of pesticides distribution on diseased or susceptible areas of the crop plants.

Among possible sensing technics for disease symptoms detection, proximal optical sensing has specific characteristics especially relevant for field applications on grapevine and other specialty tree-crops. In particular, it can inspect the vertical structure of the canopy, allowing for potential on-the-go detection of early symptoms even at centimeter/sub-centimeter scale.

The possibility to optically detect disease symptoms relies on the modifications induced by the pathogen in the plant tissue and, in turn, in the way how light interacts with it. Beside disease-specific pigmentation, main optical effects of plant diseases are associated to spectral absorption bands of chlorophyll, where tissue degradation induced by pathogens is especially emphasized.

As case studies we consider here the fully automatic selective spraying of powdery mildew diseased areas on grapes leaves as they are encountered in vineyard conditions. This paper reports on first results of a session of greenhouse experiments, with the objective of identifying disease foci within healthy canopy based on optical sensing and by sensing feedback to position a precision spraying actuator by the manipulator to selectively and accurately apply pesticides solely onto infected areas.

\section{The robot system}

\section{Robotic manipulator}

The manipulator was designed for agricultural applications in selective harvesting and precision spraying. The selective harvesting task has the highest requirements on the dexterity and accuracy in positioning of the end-effector tool. Thus, this application was the basis for the kinematic and mechatronic design, resulting in a nine degrees-offreedom (DoFs) manipulator. For further details on the design refer to Baur et al. (2012).

For the precision spraying application, the end-effector must be roughly positioned at a distance of $0.4-0.6 \mathrm{~m}$ in front of the canopy and dealing with a canopy height of about $0.9 \mathrm{~m}$. Furthermore, the endeffector should be able to spray on a target area from several directions to improve the spray coverage. Since the sprayer is rotationally symmetric, only the rotations about the $\mathrm{x}-$ and $\mathrm{z}-$ axis (cf. Figure 2) are relevant. Due to the modular design, the nine DoFs manipulator can be reconfigured to a six DoFs manipulator (the kinematic scheme is shown in Figure 1) which is more suitable for this application.

For having the manipulator ready to work under greenhouse conditions, it was necessary to build an extra cover to protect the robot against spray droplets which in addition to the high humidity conditions in the environment could lead to a short circuits on the electronics. As a results, a hard cover encloses the carriage of the linear bearing while a flexible shell protects the subsequent five joints. The casing is built up of several pieces of polyoxymethylen which are glued to two main parts. One part covers the backside, the other part is fixed at the front and serves as intersection to the soft cover. This shell is welded together out of four pieces of ripstop-nylon which is coated with a film of Thermoplastic polyurethane. The resulting tube can be wear on the arm, fixed at the sliding and is finished with an angle bracket, which is fastened to the manipulator. The bracket serves both as mounting point and for the connection of a waterproof plug. Thus power and data signals for the end effector are provided from within the protection.

A microcontroller interface board (Atmel ${ }^{\circledR}$ AT90CAN32) which provides several digital and analog IO's for various end-effectors (precision sprayer, apple gripper, and sweet-pepper fruit removal unit) as well as a CAN interface to the manipulator real-time control unit was designed. Thus, the nozzle as well as the fan speed of the precision sprayer can be controlled by simple ROS (Open Source "Robot Operating System“ by Willow Garage, http//www.ros.org) messages.

For the positioning, the goal coordinates of the tool-center-point of the sprayer are send to the real-time control unit via the ROS interface

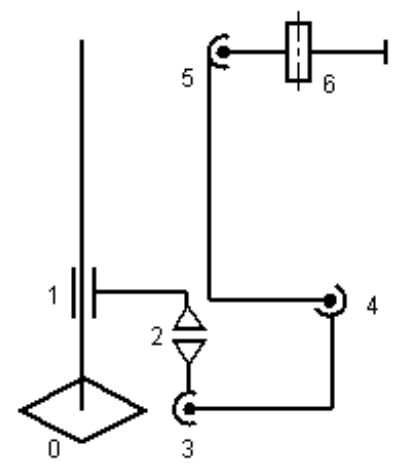

Figure 1. Kinematic scheme of the manipulator in spraying configuration

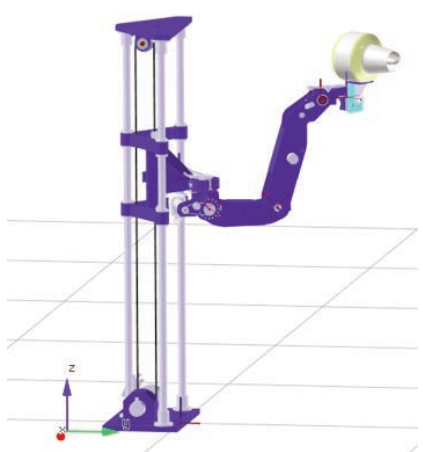

Figure 2. Visualization of the manipulator equipped with the spraying end-effector.

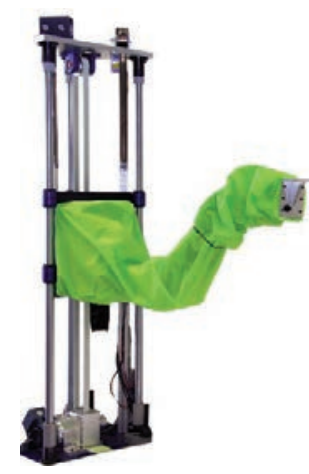

Figure 3. Manipulator System with waterproof cover. 
(cf. Figure 4). The inverse kinematics is computed on the real time PC on the velocity level (Siciliano (2009)). To avoid instability of this computation close to kinematic singularities, a configuration dependent damping factor is added. This results in a stable, but less accurate solution of the inverse kinematics close to kinematic singularities. The computed joint trajectory is than sent to the low level motor controllers of the manipulator in each time step.

\section{Disease sensing}

Disease sensing was based on multispectral image analysis of canopy under diffuse illumination. All the equipment was installed in a dockable aluminum frame which contained an RGB color camera (acA1300, Basler, Germany), an R-G-NIR multispectral camera (MS4100, DuncanTech, USA), a data acquisition PC, panels for diffuse illumination of the imaged area and power supply. The cameras were mounted on a sliding structure allowing position adjustments in height. During the experiments the cameras position was kept constant at an height of 1,4 m. To provide background regularization and avoid multiple viewing across glass walls in the greenhouse, a black, low-reflective shield was positioned on the back of the imaged plants.

For disease detection only R-G-NIR multispectral camera was used. The multispectral camera acquires $1912 \times 1076$ pixels, 8 bit images in three distinct spectral channels: green $(540 \mathrm{~nm})$, red $(660 \mathrm{~nm})$ and near infrared $(800 \mathrm{~nm})$. The area imaged was about $1.0 \mathrm{x} 0.5 \mathrm{~m}$, resulting in a spatial resolution of about $0.5 \mathrm{~mm} /$ pixel. At each acquisition, raw pixels intensity in each channel was normalized using reflectance standard panels (Spectralon 20\%, 50\% and 99\%, Labsphere, USA) kept in the field of view of the camera. Disease symptoms identification is based on the combination of two approaches: one based on the value of two spectral indexes calculated at pixel level, and the other based on relative variations (local gradients) of grey level intensity in the red channel.

A fairly clear discrimination between healthy and diseased areas can be obtained by considering for each pixel the pair of values obtained with the two spectral indexes:

$$
\begin{aligned}
& \mathrm{I} 1=\operatorname{Red} /(\operatorname{Red}+\text { Green+NIR }) \\
& \mathrm{I} 2=\left(\operatorname{Red}^{*} \text { Green }\right) / \mathrm{NIR}^{\wedge} 2
\end{aligned}
$$

These indexes are designed to capture the reflectance variations in either red or green channel, or a combination of both, related to chlorophyll absorption band which is especially expected to respond to local tissue degradation linked to a pathogen attack (Oberti et al., 2012).

Nevertheless, disease classification results based only on (I1, I2) combinations appears to be prone to significant false positive detections (i.e. non diseased areas classified as diseased). This was found to be especially related to specular reflections on glossy leaf tissue and to specific structures as leaf veins or young, green branches. To reinforce the classification robustness, a local approach was adopted, based on gradient intensity of pixel levels in the red spectral band, allowing to discriminate between smooth changes in intensity and sharp changes more likely due to reflections effects.

The two approaches are integrated within a classifier and when the two methods agree above a certain probability threshold, a set of binary regions in the image. After morphological filtering aimed to remove isolated pixels and small areas, regions left are then assumed as diseased tissue, while the rest of the foreground is assumed to correspond to healthy tissue.

\section{Sprayer end effector}

The sprayer end effector consists of an axial fan as airflow generator, an airflow duct, a pesticide nozzle with anti-dripping device, an electrical connector for power supply and control signals, all enclosed in a plastic chassis. The sprayer end effector was designed with the main goal of performing precision spot spraying of small patches of infected areas. In operation the end-effector has to be connected to a pumping station and a pesticide formulation tank placed at the base of the manipulator. The pesticide delivery flow-rate ranges from $15 \mathrm{ml} / \mathrm{min}$ to $50 \mathrm{ml} / \mathrm{min}$, depending on the selected nozzle tip. The one used during the experiments has a flow-rate of $30 \mathrm{~mL} / \mathrm{min}$ at a pressure of 4 bars, with a full cone pattern of $30^{\circ}$ and an average diameter of spray droplets of about $150 \mu \mathrm{m}$. The fan features PWM control for rotational speed, allowing to set the air-carrier flow velocity from 5 to $30 \mathrm{~m} / \mathrm{s}$. As overall result, the sprayer end effector delivers a circular spraying pattern of a constant diameter of $0.15-0.2 \mathrm{~m}$ over a wide range of spraying distances $(0.4-1.5 \mathrm{~m})$.

\section{Greenhouse experiments}

\section{Plant material preparation and canopy setup}

Selective spot spraying concept was tested in a session of greenhouse experiments conducted in early 2013 on grapevine canopy with localized symptoms of powdery mildew (Erysiphe necator), one of the major diseases for this specialty crop.

To this aim in spring 2012, 180 plants of Vitis vinifera L. cv Cabernet Sauvignon were propagated from wood, and nursed in $30 \mathrm{~cm}$ diameter pots by maintaining greenhouse environment under controlled conditions at $25 / 20{ }^{\circ} \mathrm{C}$ day/night temperature, with $50-75 \%$ relative humidity and a 16 -h photoperiod $\left(40 \mu\right.$ mol quanta $\left.\mathrm{m}^{-2} \mathrm{~s}^{-1}\right)$. Grapevine plants were pruned in autumn so that they reached full development stage in February 2013. This schedule enabled off-season use of the CROPS manipulator after field experiments on other agricultural operations in other sites considered within the project.

A subset of grown grape plants was inoculated by brushing Erysiphe necator conidia onto the adaxial (upper face) epidermis of healthy leaves in order to induce powdery mildew infection. The infected plants were nursed in a separated greenhouse room where favourable environmental conditions for disease development were maintained.

For purpose of experimenting the selective spraying system, the plant material was arranged in a greenhouse setup in order to simulate vineyard canopy conditions by aligning healthy grapevine plants in pots on tables (Figure 4, right). Within the recreated canopy wall, diseased plants with different levels of symptoms were positioned in order to simulate the presence of localised disease foci within healthy vegetation, representing the actual targets of selective spot spraying to be performed by CROPS robot.

Different replicates of grapevine canopy plot (5 m in length $x 1,8 \mathrm{~m}$ in height) were obtained by preparing different plants arrangements by substituting healthy and diseased plants with other spare samples and/or changing their position in the line.

Prior to each robotic spraying pass, the canopy plot was accurately monitored by visual inspection by a plant Pathologist. Position, size and intensity of disease foci symptoms were recorded and used for assessing the results obtained with each robotic spraying treatment.

\section{Robotic system setup and experimental procedure}

For the spot spraying experiments session, the disease detection system and the manipulator equipped with precision spraying actuator were integrated on a trailer platform, hosting the PC for data acquisition and real-time processing and the controller of the manipulator. The manipulator geometrical coordinates system was then registered 
with the coordinates system of the multispectral camera. With this procedure, the position of a point at a known frontal distance $\mathrm{X}$ from the camera and having coordinates (yi, zi) in an acquired image, can univocally translated in a vector of coordinates (xm, ym, zm) of the manipulator reference system.

During the experiments, the trailer holding the robotic system was positioned frontally to the recreated canopy and while traveling was kept at a constant distance from the midline of the vegetation. The trailer was moved in front of the canopy wall at steps of $10 \mathrm{~cm}$. At each position a multispectral image of the canopy was acquired and processed in real-time.

The obtained results in terms of presence and position of disease symptoms were written in to a spraying targets queue file. Concurrently, the targets in the queue list identified by previous acquisitions and having a position in the canopy reachable by the manipulator at current trailer standing, were aggregated in single spray spots (i.e. within circles having a diameter of $15 \mathrm{~cm}$ ). The coordinates of the center of each resulting spray spot were then passed to the manipulator controller through ROS messages. The corresponding targets were assumed treated and consequently removed from the queue list.

In order to maximize the homogeneity of spraying deposit and covering of targets, each single spot (i.e. a circular area of the canopy with a diameter of $15 \mathrm{~cm}$ ) was sprayed from three different directions. For each spot to be sprayed the manipulator was then commanded to bring the end effector in three positions from where the nozzle was operated to deliver one third of the nominal flow ( $0.5 \mathrm{~s}$ on a total of $1.5 \mathrm{~s}$ of spraying time). The sequence of three positions was: i) distance from spot's center $d=0.6 \mathrm{~m}$, latitude angle $\alpha=30^{\circ}$, longitude angle $\gamma=0^{\circ}$; ii) distance from spot's center $\mathrm{d}=0.6 \mathrm{~m}$, latitude angle $\alpha=-30^{\circ}$, longitude angle $\gamma=+30^{\circ}$; iii) distance from spot's center $d=0.6 \mathrm{~m}$, latitude angle $\alpha=-30^{\circ}$, longitude angle $\gamma=-30^{\circ}$.

With this spraying sequence timing, each identified target received a corresponding application rate of $375 \mathrm{~L} / \mathrm{ha}_{\text {foliage }}$ which is a representative value of current grapevine protection treatments.

After all the targets in queue falling in the workspace of the manipulator at current trailer standing were sprayed, a message of duty accomplished was generated and trailer was then moved by $10 \mathrm{~cm}$ to next step.

In order to assess the performance of the automatic disease detection system the algorithm output for each robotic pass was compared with the visual inspection records (position, size, intensity of symptoms) made by plant Pathologist. The final spray deposit on the canopy, and specifically on individual disease foci, was evaluated by spraying a fluorescent dye mixture.

\section{Results}

The operative results obtained with robotic selective spraying of disease symptoms were quantitatively assessed through three parameters:

- the sensitivity of the selective treatment, which expresses the capability of real covering the targets (numerically defined as the fraction of canopy area to be sprayed which was actually sprayed by the robot);

- the specificity of the selective treatment, which expresses the capability of avoiding excess of unnecessary spraying (numerically defined as the canopy area fraction of the area not to be sprayed which was actually left unsprayed by the robot);

- the pesticide reduction of the selective treatment, which expresses the reduction of used pesticide in comparison of a conventional uni-

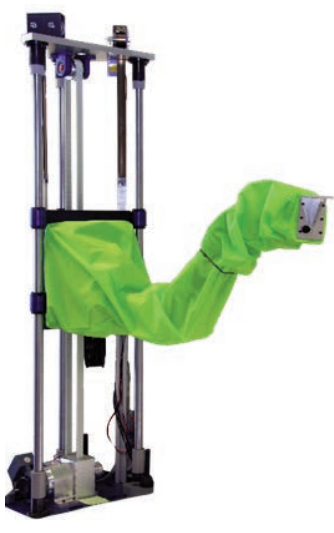

Figure 4. Hardware and software architecture of the robotic manipulator.

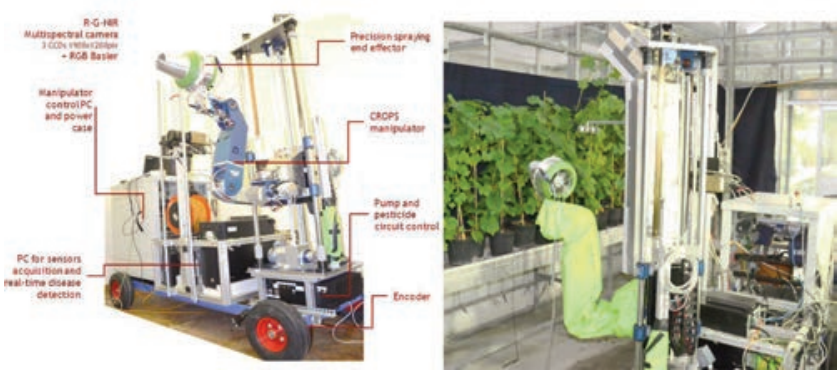

Figure 5. The robotic setup integrated on a trailer during preparation in the lab (left), and during experiments of selective spraying on grapevine

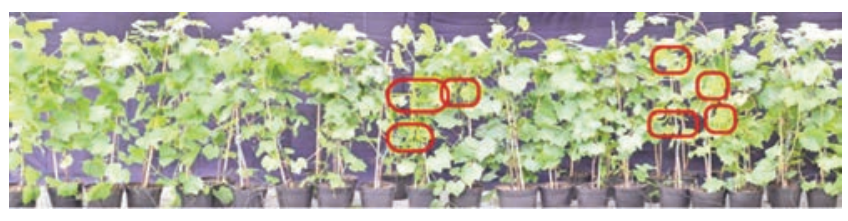

Figure 6. Example of grapevine plants setup to recreate a vineyard canopy wall in greenhouse conditions. Among aligned healthy plants, powdery mildew infected plants with different levels of disease symptoms were positioned to simulate the presence of localised disease foci (in red) within healthy vegetation.

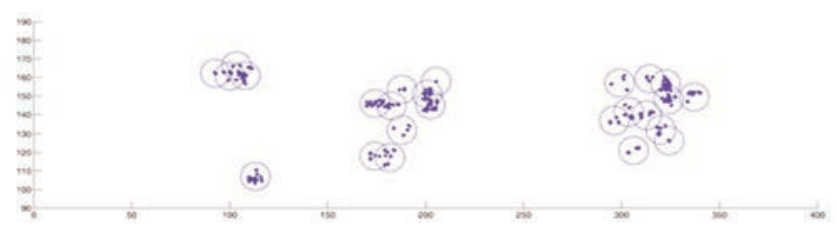

Figure 7. Example of detected diseased areas as automatically identified by the robotic system and corresponding spray spots (blue circles) commanded to the sprayer effector. There is an evident overlap with Pathologist prescription (Fig.5 as ground truth) for two foci at position around 170-200 and around 300-340, while around position 100 the robotic system has erroneously treated two false positive regions 
form spray distribution operated at the same application rate (numerically defined as the ratio of canopy area sprayed, including multiple sprayings from overlapping spots, on the area of the canopy plot).

Figure 5 shows an example of the different replicates of grapevine canopy plots prepared for the experiments. Red frames in the image indicate the disease foci positions as obtained by plant Pathologist monitoring, hence representing the actual target of selective spraying. In Figure 6, the corresponding symptoms map as automatically detected by the robotic system is shown. In the same chart, the blue circles represent the spray spots which the manipulator was commanded to apply to the canopy. It can be noticed that there is a remarkable qualitative overlap between results in Figure 6 and the plant Pathologist prescription (red frames in Figure 5) for what concerns two disease foci located around position $\mathrm{x}=170-200$ and around position $\mathrm{x}=300-340$ in the chart. On the other hand, the robotic system erroneously detected and treated two false positive regions around position $x=100$.

In this specific example the actual disease area represents the $1.6 \%$ of the total canopy area. The sensitivity of the robotic selective spraying resulted $85 \%$, while the selectivity was $92 \%$ meaning that only $8 \%$ of the total healthy area was sprayed unnecessary. The total amount of sprayed pesticide was $16 \%$ of the amount used in a conventional uniform treatment, with a remarkable reduction of $84 \%$.

Even if described just as an illustrative example, these results are at our best knowledge the first application of selective, intelligent treatment of plant diseases, representing a possible steps towards new developments in precision pest management and precision protection of crops.

\section{References}

Baeten J., Donné K., Boedrij S., Beckers W., Claesen E. 2008. Autonomous fruit picking machine: A robotic apple harvester, Tracts in Advanced Robotics, Vol. 42, pp. 531-539.

Baur J., Pfaff J., Ulbrich H., Villgrattner T. 2012. Design and development of a redundant modular multipurpose agricultural manipulator, Advanced Intelligent Mechatronics (AIM), 2012 IEEE/ASME International Conference on', Kaohsiung, Taiwan, 823-830.

Guo J., Zhao D., Ji W., Xia W. 2010. Design and control of the open apple-picking-robot manipulator, Proc. IEEE Int. Conf. on Computer Science and Information Technology (ICCSIT), Vol. 2, pp. 5-8.

Katupitiya J., Eaton R., Cole A., Meyer C., Rodnay G. 2005. Automation of an Agricultural Tractor for Fruit Picking, in Proc. IEEE Int. Conf. on Robotics and Automation (ICRA), pp. 3201-3206.

Kitamura S., Oka, K. 2005. Recognition and cutting system of sweet pepper for picking robot in greenhouse horticulture, Proc. IEEE Int. Conf. on Mechatronics and Automation (ICMA), Vol. 4, pp. 18071812.

Oberti R., Tirelli P., Marchi M., Calcante A., Iriti M., Borghese N.A. 2012. Automatic diseases detection in grapevine under field conditions. Proc. of the first International Conference on Robotics and associated High-technologies and Equipment for agriculture applications. 2012, p. 101-106, Pisa University Press, ISBN: 9788867410217

Siciliano, B.; Sciavicco, L. \& Villani, L., 2009. Robotics: modelling, planning and control, Springer Verlag. 